\title{
EFEITO DA FUNÇÃO DE MAPEAMENTO NA DISTRIBUIÇÃO DA VELOCIDADE DA FASE LÍQUIDA NA SEÇÃO DE UMA COLUNA DE BOLHAS POR PIV
}

\author{
R. L. AMARAL ${ }^{1}$, D. I. S. FORERO ${ }^{1}$, K. K. da $\operatorname{COSTA}^{1}$, O. P. TARANTO ${ }^{1}$, S.S.V. \\ VIANNA $^{1}$, M. MORI $^{1}$. \\ ${ }^{1}$ Universidade Estadual de Campinas, Faculdade de Engenharia Química. \\ E-mails para contato: rodrigo.amara101@hotmail.com,val@feq.unicamp.br, \\ savio@feq.unicamp.br,mori@feq.unicamp.br
}

\begin{abstract}
RESUMO - Aplicações da técnica de medida PIV (Velocimetria por Imagem de Partícula) em escoamentos dentro de equipamentos industriais, frequentemente com geometrias complexas, exige uma resolução espacial precisa no processamento de imagem. Um bom exemplo pode ser encontrado na investigação da fluidodinâmica em uma coluna de bolhas. A existência de curvaturas na coluna produz deformações locais interferindo na qualidade da medida PIV. Uma maneira de evitar esse problema é relacionar o domínio espacial com o domínio de imagem, na calibração, através de uma função de mapeamento, compensando as deformações de qualquer janela óptica ou ambientes com índices de difração diferentes. Esse trabalho tem como objetivo investigar o efeito da função de mapeamento PIV na distribuição da velocidade da fase líquida na seção de uma coluna de bolhas. Os resultados mostram uma diferença local entre a distribuição da velocidade sem e com a função de mapeamento.
\end{abstract}

\section{INTRODUÇÃO}

As colunas de bolhas são amplamente usadas em processos químicos por fornecerem várias vantagens em relação à transferência de calor e de massa, por exemplo. Por ser uma técnica não intrusiva, o estudo hidrodinâmico e do comportamento das bolhas por PIV tem se tornado comum atualmente. A essência da imagem PIV é mapear precisamente a localização de cada partícula no plano de imagem (Figura 1a). O mapeamento deve ser uma função que relacione a localização tridimensional da partícula no fluido com o espaço bidimensional do plano de imagem (ADRIAN e WESTERWEEL, 2011). Idealmente, deseja-se encontrar uma função de mapeamento $M$ tal que para um ponto qualquer $\mathbf{X}^{\prime}=\left(\mathrm{X}^{\prime}, \mathrm{Y}^{\prime}, \mathrm{Z}^{\prime}\right)$, no plano objeto, sua localização correspondente $\mathbf{x}=(\mathrm{X}, \mathrm{Y})$, no plano de imagem, seja determinado com precisão absoluta. Na realidade, não se pode determinar experimentalmente uma função $\mathrm{M}$ com um erro igual a zero para uma geometria arbitrariamente complexa. Assim, uma estimativa para a função de mapeamento, que tem uma representação analítica, é buscada. Existem várias maneiras de determinar essa função. Por exemplo, em um procedimento de calibração, pode-se utilizar o método dos mínimos quadrados para determinar um M que aproxima os dados minimizando o erro médio quadrático (SOLOFF et al, 1997). 
a. Espaço Físico

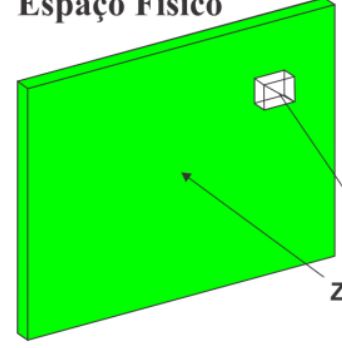

Plano de Luz

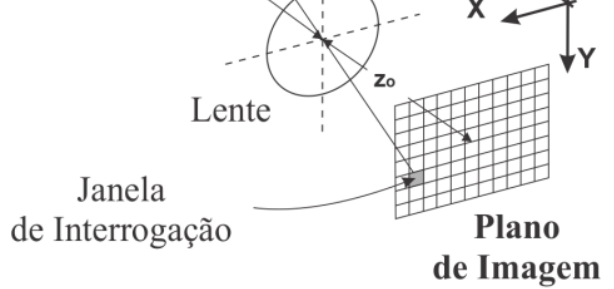

b.
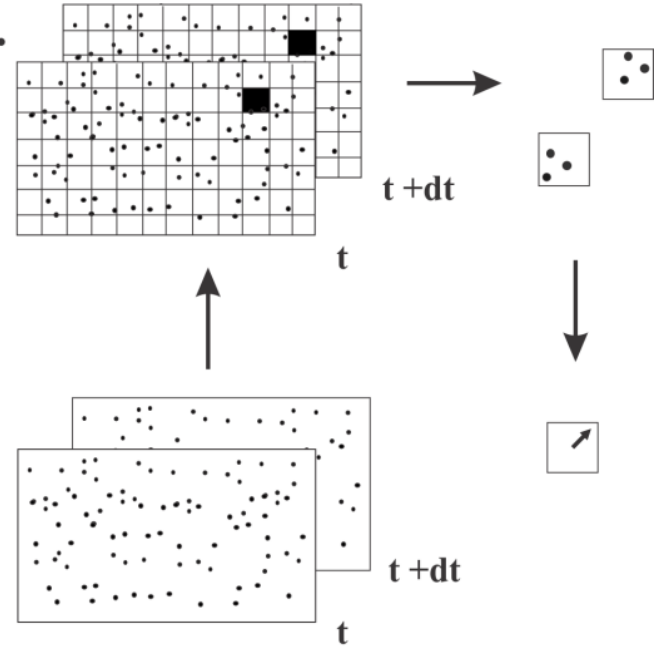

Figura 1 - (a) Representação esquemática da imagem geométrica e da (b) correlação cruzada PIV (Adaptado de Amaral, 2013).

O processo de calibração do sistema é realizado manualmente pela orientação prévia das linhas de visão das câmeras, ajustando-se a lente da câmera, certificando que a imagem completa esteja em foco, tendo como alvo uma placa de calibração. O desenho do alvo de calibração é a parte mais importante da realização deste procedimento. Em geral, diferentes experimentos podem exigir diferentes alvos de calibração. As imprecisões na calibração são causadas por distorção óptica devido ao alinhamento óptico impreciso, imperfeição no design da lente, refração por janelas ópticas, interfaces de fluidos e outros elementos de óptica em um experimento (SOLOFF et al, 1997). A função de mapeamento é utilizada no cálculo da correlação cruzada (Figura 1b).

Esse trabalho tem como objetivo investigar o efeito da função de mapeamento PIV na distribuição da velocidade da fase líquida na seção de uma coluna de bolhas.

\section{EXPERIMENTAL}

\subsection{Coluna de bolhas e sistema PIV}

A Figura 2 a apresenta o esquema da coluna de bolhas e do sistema PIV utilizado. A seção investigada esta localizada a $55,2 \mathrm{~cm}$ do distribuidor e possui um tamanho igual a 14,5 x $10 \mathrm{~cm}$ (Figura 2a e Figura 2b). A coluna é de acrílico com 14,5 cm de diâmetro, $1 \mathrm{~m}$ de altura e foi preenchida com uma altura inicial de $70 \mathrm{~cm}$ utilizando água a $25^{\circ} \mathrm{C}$. Para a fase gasosa, foi utilizado ar com uma vazão de $1 \mathrm{~L} / \mathrm{min}$. Como distribuidor, foi utilizada uma placa plana com 21 furos (com $1 \mathrm{~mm}$ de diâmetro) igualmente distribuídos na área da seção transversal da coluna em um arranjo quadrado (Figura 2c). Para que o ar inserido no escoamento possua uma melhor distribuição, o espaço imediatamente anterior ao distribuidor foi preenchido com esferas de vidro com diâmetro médio de $2 \mathrm{~mm}$. 


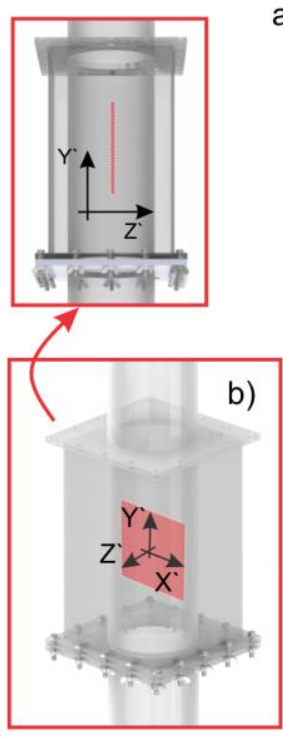

c)

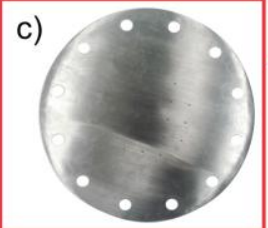

Figura 2 - (a) Coluna, sistema PIV e caixa com a fenda. (b) Área de investigação. (c)

Distribuidor da fase gasosa com 21 furos. (d) Arranjo do sistema PIV na coluna.

O sistema PIV utilizado foi desenvolvido pela LaVision e possui dois sensores CCD (1376 x 1040 e 1376 x 1024 pixels $)$ e um sistema laser Nd:YAG $(200 \mathrm{~mJ} /$ pulso e $\lambda=532$ $\mathrm{nm})$. As câmeras e o laser foram controlados com uma unidade de tempo programável (PTU) pelo software DaVis 7.2. Foram utilizadas as câmeras com um deslocamento angular de 90 (câmera A) e 80 (câmera B) graus em relação à folha de luz laser (Figura 2d). Foi utilizada em cada câmera uma lente objetiva da Nikon com distância focal de $60 \mathrm{~mm}$ (F/2.8D). Foram utilizadas Rodamina B como partícula traçadora e um filtro passa-alta, na câmera, que só permite a passagem da luz emitida pela Rodamina $(\mathrm{dp}=20-50 \mu \mathrm{m}$ e $\lambda=620 \mathrm{~nm})$. Foram utilizadas no laser duas lentes com distância focal de -10 e -20 para expandir o feixe de luz laser e uma caixa com a fenda de $1 \mathrm{~mm}$ de espessura, localizada a 1,20 $\mathrm{m}$ do laser (Figura 2d), com o objetivo de formar um plano de luz laser com espessura definida de $1 \mathrm{~mm}$ e com um perfil de intensidade homogêneo (Top-Hat).

Foram gravadas 2500 imagens com uma frequência de $4 \mathrm{~Hz}$. O tempo inter frame foi de $1500 \mu$ s produzindo um deslocamento máximo de imagem de partícula de 15 - 20 pixels. Depois da gravação das imagens, foi feito o pré-processamento e a correlação cruzada para determinação da distribuição da velocidade do líquido. Na correlação cruzada, foi utilizada uma estratégia de interrogação com dois passos sendo o primeiro com um tamanho de 128 pixels (sobreposição de $50 \%$ das janelas adjacentes) e o segundo com 64 pixels (sobreposição de $75 \%$ das janelas adjacentes). No pós-processamento, foi usado o teste da mediana de Westerweel e Scarano (2005) com um limite de remoção igual a 2 e uma vizinhança de 3 x 3 pixels. Foi feito um experimento com (experimento 1) e sem (experimento 2) uma caixa de acrílico externa preenchida com o mesmo fluido do interior da coluna, água a $25^{\circ} \mathrm{C} \mathrm{em}$ volta 
da área de investigação (Figura 2a). Essa caixa tem como função diminuir o efeito das distorções ocorridas pela curvatura da coluna cilíndrica. $\mathrm{O}$ ajuste dos modelos foi feito no software Davis 7.2.

\subsection{Função de Mapeamento e Calibração}

Pinhole: $\mathrm{O}$ modelo geométrico pinhole, utilizado pela maioria das câmeras, é baseado no teorema da interseção de linhas (Figura 1a) entre o espaço físico e o plano de imagem pelo ajuste dos parâmetros físicos como, por exemplo, distância focal da lente (f), translação (T) e rotação $(\mathrm{R})$ do objeto $\left(\mathrm{x}_{0}, \mathrm{y}_{0}\right)$ nos eixos $\mathrm{X}^{\prime}, \mathrm{Y}^{\prime}$ e $\mathrm{Z}^{\prime}$ (Equação 1). Hartley e Zissermann (2000) detalham toda a reconstrução geométrica do modelo pinhole.

$$
\mathbf{X}=\mathrm{M}\left(f ; \mathrm{T}_{x, y, z} ; \mathbf{R}_{\mathrm{x}, \mathrm{y}, \mathrm{z}}\right) \cdot \mathbf{X}^{\prime}
$$

Polinomial de terceira ordem: Para cada um dos planos de calibração, a relação entre as coordenadas físicas $\left(\mathrm{X}^{\prime}, \mathrm{Y}^{\prime}, \mathrm{Z}^{\prime}\right)$ e as coordenadas de imagem $(\mathrm{X}, \mathrm{Y})$ foi descrita por um ajuste polinomial de terceira ordem dada pela Equação 2, em que $\mathrm{dX}^{\prime}$ e $\mathrm{dY}^{\prime}$ são definidos na Equação 3. Os deslocamentos $\mathrm{dX}^{\prime}$ e $\mathrm{dY}^{\prime}$ são determinados usando as coordenadas normalizadas $\mathrm{s}=2\left(\mathrm{X}-\mathrm{X}_{0}^{\prime}\right) / \mathrm{n}_{\mathrm{x}}$ e $\mathrm{t}=2\left(\mathrm{Y}-\mathrm{Y}^{\prime}{ }_{0}\right) / \mathrm{n}_{\mathrm{y}}$, em que $\mathrm{nx}$ e ny é o tamanho da imagem em pixel e $\left(\mathrm{X}_{0}^{\prime}, \mathrm{Y}^{\prime}{ }_{0}\right)$ da escala no frame (LaVision, 2005).

$$
\begin{aligned}
& \left(\begin{array}{l}
X \\
Y
\end{array}\right)=\left[\begin{array}{l}
X^{\prime}+d X\left(X^{\prime}, Y^{\prime}\right) \\
Y^{\prime}+d Y\left(X^{\prime}, Y^{\prime}\right)
\end{array}\right] \\
& \left(\begin{array}{l}
d X^{\prime} \\
d Y^{\prime}
\end{array}\right)=\left(\begin{array}{l}
a_{0}+a_{1} s+a_{2} s^{2}+a_{3} s^{3}+a_{4} t+a_{5} t^{2}+a_{6} t^{3}+a_{7} s t+a_{8} s^{2} t+a_{9} s t^{2} \\
b_{0}+b_{1} s+b_{2} s^{2}+b_{3} s^{3}+b_{4} t+b_{5} t^{2}+b_{6} t^{3}+b_{7} s t+b_{8} s^{2} t+b_{9} s t^{2}
\end{array}\right)
\end{aligned}
$$

Placa de calibração: Foi utilizada uma placa feita de Polionda® para a calibração. Na placa, foram feitos pontos, com um diâmetro (d) de $0,5 \mathrm{~mm}$, espaçados (l) a 2,5 mm (arranjo quadrado) entre si (Figura 3.9a). Esses parâmetros foram escolhidos de acordo com o tamanho do campo de visão das câmeras (Seção 3.2.1) e com a distorção causada pelo tubo. A estimativa do número de pontos da placa é proporcional ao tamanho do campo de visão e a distância entre os pontos é inversamente proporcional à quantidade de distorção da imagem causada pelo tubo. A Figura 3 b apresenta a identificação dos pontos da placa pelo Davis 7.2. Com a identificação dos pontos pelo software, nessa resolução do sistema de imagem (12 pixel $\mathrm{s} / \mathrm{mm}$ ), percebe-se que a escolha do espaçamento $1=2,5 \mathrm{~mm}$ é boa. $\mathrm{O}$ ajuste da função da função de mapeamento pelo Davis é apresentando na Figura 3c. 
a.

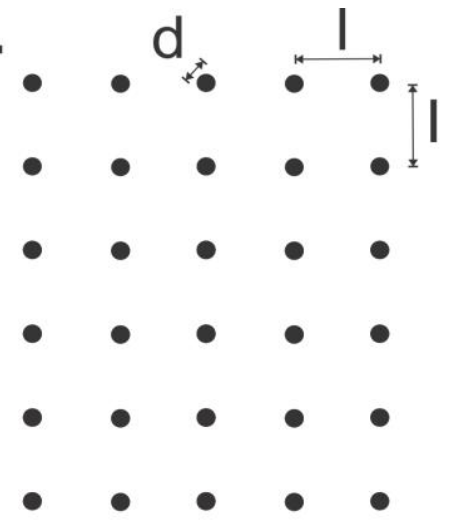

b.

\begin{tabular}{|c|c|c|c|}
\hline 田 & 州 & 田 & \\
\hline 田 & 田 & 田 & $E$ \\
\hline 田 & 田 & 田 & \\
\hline 田 & 田 & 田 & \\
\hline 4 & 田 & 田 & \\
\hline$\overline{7}$ & 用 & 用 & \\
\hline
\end{tabular}

C.

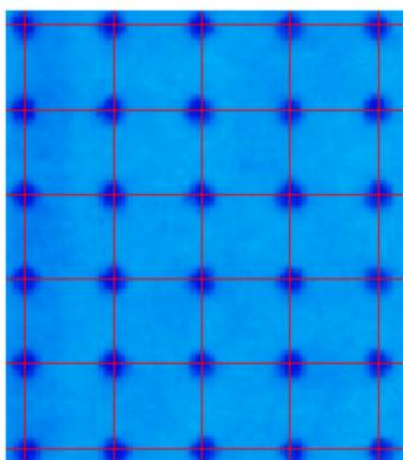

Figura 3 - (a) Pontos da placa de calibração $(1=2,5 \mathrm{~mm}$ e d =0,5 mm). (b) Identificação dos pontos e (c) ajuste da função de mapeamento (linhas vermelhas) pelo software Davis 7.2

\section{RESULTADOS}

A Tabela 1 apresenta o erro de ajuste do modelo pinhole e polinomial das câmeras A e B para os experimentos 1 e 2. Percebe-se que o modelo polinomial se ajustou melhor que o pinhole ao apresentar os menores erros. Segundo LaVision (2005), um erro menor que 1 pixel é bom, um valor menor que 0,3 pixel é excelente e um valor maior que 2 pixels é questionável. O ajuste pelo modelo pinhole é aceitável para as câmeras A e B no experimento 1. Sem o uso da caixa com água, o experimento 2 apresentou os maiores erros, acima de 2 pixels, para os dois modelos, causado pela distorção da curvatura do tubo.

Tabela 1 - Erro de ajuste do modelo pinhole e polinomial de terceira ordem das câmeras A e B para os experimentos 1 e 2

\begin{tabular}{|c|c|c|c|c|c|c|c|c|}
\hline Modelo & \multicolumn{2}{|c|}{ Pinhole } & \multicolumn{2}{c|}{ Polinomial } & \multicolumn{2}{c|}{ Pinhole } & \multicolumn{2}{c|}{ Polinomial } \\
\hline Câmera & A1 & B1 & A1 & B1 & A2 & B2 & A2 & B2 \\
\hline $\mathbf{N}^{\mathbf{0}}$ de pontos & 2492 & 2528 & 2492 & 2528 & 2211 & 2279 & 2211 & 2279 \\
\hline Erro (pixel) & 0,805 & 1,113 & 0,579 & 0,589 & 4,448 & 10,648 & 2,874 & 3,225 \\
\hline
\end{tabular}

Os parâmetros dos modelos pinhole e polinomial para cada câmera nos dois experimentos são apresentados nas Tabelas 3, 4 e 5. Percebe-se na Tabela 3 que os parâmetros físicos estimados distância focal e translação, que se aproximaram do real foi na câmera A e B do experimento 1 (A1 e B1), que apresentou os menores erros.

Tabela 2 - Parâmetros do modelo pinhole

\begin{tabular}{|c|c|c|c|c|}
\hline Câmera & Distância focal $\boldsymbol{f}$ [mm] & $\mathbf{x}_{\mathbf{0}}$ [pixel] & $\mathbf{y}_{\mathbf{0}}$ [pixel] & Resolução [pixels $/ \mathbf{m m}$ ] \\
\hline A1 & 31,128 & 708,806 & 481,440 & 9,614 \\
\hline B1 & 79,892 & 689,504 & 497,767 & 9,131 \\
\hline A2 & $-9,658$ & 777,314 & 674,143 & 12,044 \\
\hline B2 & 738,022 & 757,427 & 659,146 & 11,128 \\
\hline
\end{tabular}


Tabela 3 - Parâmetros do modelo pinhole (Continuação)

\begin{tabular}{|c|c|c|c|c|c|c|}
\hline \multirow{2}{*}{ Câmera } & \multicolumn{4}{|c|}{ Posição da placa de calibraçãa } \\
\cline { 2 - 8 } & \multicolumn{3}{|c|}{ Translação [mm] } & \multicolumn{3}{|c|}{ Rotação $\left.^{[\mathbf{0}}\right]$} \\
\hline \multirow{2}{*}{ A1 } & $\mathbf{T x}$ & $\mathbf{T z}$ & $\mathbf{T y}$ & $\mathbf{R x}$ & $\mathbf{R z}$ & $\mathbf{R y}$ \\
\hline B1 & $-2,636$ & 6,639 & 515,067 & 2,632 & 0,045 & 0,091 \\
\hline $\mathbf{A 2}$ & 3,085 & 5,454 & 1358,500 & 2,107 & 10,925 & 0,554 \\
\hline B2 & 3,553 & 1,688 & 10266,400 & 37,228 & $-0,217$ & 1,084 \\
\hline
\end{tabular}

Tabela 4- Parâmetros do modelo polinomial: $\mathrm{X}_{0}$ e $\mathrm{Y}_{0}$

\begin{tabular}{|c|c|c|c|c|c|}
\hline Câmera & $\mathbf{X}_{\mathbf{0}}$ & $\mathbf{n}_{\mathbf{x}}$ & $\mathbf{Y}_{\mathbf{0}}$ & $\mathbf{n}_{\mathbf{y}}$ & Tamanho da imagem deformada [pixels $\mathbf{2}^{\mathbf{}}$ ] \\
\hline $\mathbf{A 1}$ & 706,621 & 1376 & 477,244 & 1040 & $1425 \times 1073$ \\
\hline $\mathbf{B 1}$ & 712,19 & 1376 & 512,867 & 1024 & $1462 \times 1077$ \\
\hline $\mathbf{A 2}$ & 775,13 & 1376 & 649,624 & 1040 & $1492 \times 1398$ \\
\hline $\mathbf{B 2}$ & 731,28 & 1376 & 647,232 & 1024 & $1441 \times 1319$ \\
\hline
\end{tabular}

Tabela 5- Coeficientes do ajuste da função de mapeamento pelo modelo polinomial para as câmeras A e B dos experimentos 1 e 2.

\begin{tabular}{|c|c|c|c|c|c|c|c|c|c|c|}
\hline Câmera & $\mathbf{a}_{\mathbf{0}}$ & $\mathbf{a}_{\mathbf{1}}$ & $\mathbf{a}_{\mathbf{2}}$ & $\mathbf{a}_{\mathbf{3}}$ & $\mathbf{a}_{\mathbf{4}}$ & $\mathbf{a}_{\mathbf{5}}$ & $\mathbf{a}_{\mathbf{6}}$ & $\mathbf{a}_{\mathbf{7}}$ & $\mathbf{a}_{\mathbf{8}}$ & $\mathbf{a}_{\mathbf{9}}$ \\
\hline $\mathbf{A 1}$ & 24,811 & 15,189 & $-0,298$ & 4,290 & $-1,656$ & $-0,806$ & 1,647 & $-3,972$ & $-0,234$ & 0,345 \\
\hline $\mathbf{B 1}$ & 48,094 & 34,435 & $-6,203$ & 2,492 & $-2,423$ & $-0,038$ & 1,456 & $-3,494$ & $-0,057$ & 0,056 \\
\hline $\mathbf{A 2}$ & 53,824 & $-9,467$ & 7,353 & 47,821 & $-9,953$ & $-0,463$ & 1,107 & $-2,219$ & 1,495 & $-0,095$ \\
\hline $\mathbf{B 2}$ & $-7,923$ & $-33,575$ & 37,939 & 53,966 & $-10,487$ & $-0,345$ & 1,233 & $-1,440$ & 2,449 & $-0,033$ \\
\hline $\mathbf{C a ̂ m e r a}$ & $\mathbf{b}_{\mathbf{0}}$ & $\mathbf{b}_{\mathbf{1}}$ & $\mathbf{b}_{\mathbf{2}}$ & $\mathbf{b}_{\mathbf{3}}$ & $\mathbf{b}_{\mathbf{4}}$ & $\mathbf{b}_{\mathbf{5}}$ & $\mathbf{b}_{\mathbf{6}}$ & $\mathbf{b}_{\mathbf{7}}$ & $\mathbf{b}_{\mathbf{8}}$ & $\mathbf{b}_{\mathbf{9}}$ \\
\hline $\mathbf{A 1}$ & 16,618 & 1,252 & $-0,181$ & $-0,383$ & 12,476 & $-2,032$ & 1,530 & 0,026 & 1,324 & $-0,053$ \\
\hline $\mathbf{B 1}$ & 32,509 & 6,510 & $-0,169$ & $-0,157$ & 16,423 & $-1,373$ & 1,279 & $-5,163$ & 1,155 & 0,054 \\
\hline $\mathbf{A 2}$ & 175,036 & 7,944 & $-6,284$ & $-0,568$ & 120,916 & $-0,904$ & 0,762 & 0,990 & 4,304 & 0,055 \\
\hline $\mathbf{B 2}$ & 155,826 & 11,903 & $-5,412$ & 1,093 & 97,388 & $-0,644$ & 0,754 & $-2,489$ & 5,127 & 0,107 \\
\hline
\end{tabular}

A Figura 4a apresenta o perfil simétrico de Vy médio para as 2500 imagens sem e com o procedimento de calibração utilizando os dois modelos na função de mapeamento. Além da diferença entre o perfil sem e com calibração, percebe-se que o perfil de Vy médio utilizando os modelos pinhole e polinomial para as duas câmeras (A e B), aparentemente, se sobrepõem. É observada uma pequena diferença entre os modelos para cada câmera no perfil de Vx médio (Figura 4b) e uma diferença considerável entre as câmeras (A e B) por conta do deslocamento. 

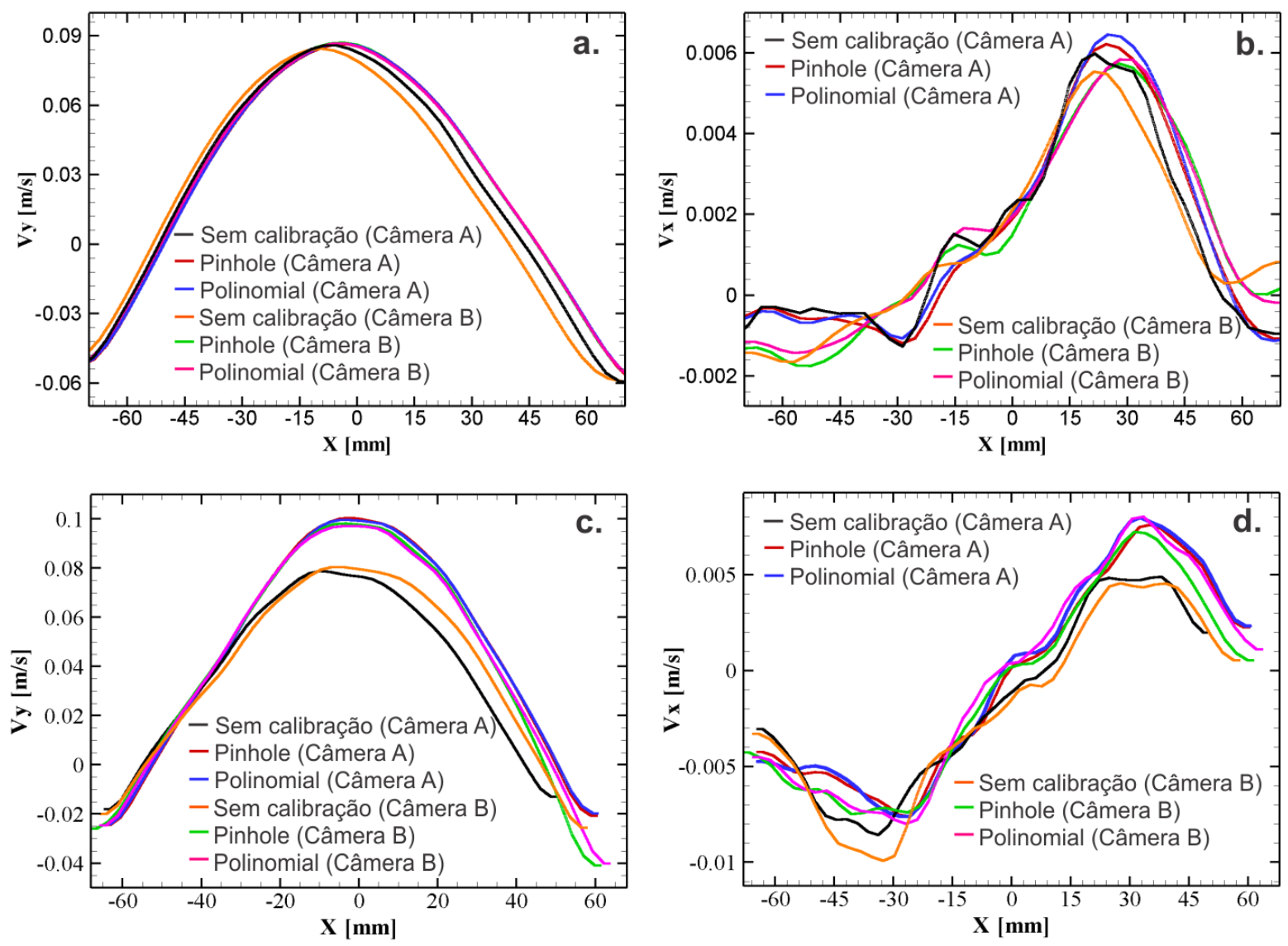

Figura 4 - Perfil de Vy e Vx médio para os experimentos 1 (a e b) e 2 (c e d), respectivamente.

No experimento 2, sem a caixa acrílica preenchida com água (Figura 4c e 4d), percebese uma perda de informação do perfil de Vy e Vx próxima as paredes do tudo por conta da forte distorção radial $(X<-60 \mathrm{~mm}$ e $X>60 \mathrm{~mm}$ ). A mesma diferença relatada no experimento 1 é observado nos perfis médio de $\mathrm{Vy}$ e $\mathrm{Vx}$ sem e com o procedimento de calibração para os dois modelos.

\section{CONCLUSÕES}

Nesse trabalho foi investigado o efeito da função de mapeamento PIV na distribuição da velocidade da fase líquida na seção de uma coluna de bolhas. Foram analisados dois modelos (pinhole e polinomial de terceira ordem) avaliando o erro de ajuste e comparando os perfis médios de Vy e Vx. Foi analisado também o efeito da caixa com água que tem como função diminuir o efeito da distorção da curvatura da coluna. Sem o uso da caixa com água, percebese uma perda de informação próxima à parede devido a curvatura da coluna. $\mathrm{O}$ modelo polinomial funcionou melhor que o pinhole nos casos em que a distorção radial é muito forte.

\section{AGRADECIMENTOS}

Os autores agradecem ao apoio financeiro da Petróleo Brasileiro S. A. - PETROBRÁS, termo de compromisso 0050.0070828.11.9, que garantiu o desenvolvimento deste trabalho e à 


\section{CAPES.}

\section{REFERÊNCIAS}

ADRIAN, R. J.; WESTWERWEEL, J. Particle Image Velocimetry. Nova York: Cambridge University Press, 2011.

AMARAL, R. L. Efeito da autocalibração volumétrica para PIV tomográfica no campo de velocidade em uma seção de um riser de um leito fluidizado circulante. Dissertação de Mestrado. FEQ-UNICAMP. Campinas-SP, 2013.

HARTLEY R, ZISSERMANN A, Multiple View Geometry in Computer Vision, Cambridge University Press, 2000.

LAVISION. Product-Manual: FlowMaster. Göttingen. 2005.

SOLOFF, S.; ADRIAN, R.; LIU, Z. Distortion compensation for generalized stereoscopic particle image velocimetry. Measurement Science and Technology, v. 8, p. 1441-1454, 1997. 